\title{
EL LICEO EXPERIMENTAL MANUEL DE SALAS: UN APORTE DE LA UNIVERSIDAD DE CHILE A LA EDUCACIÓN NACIONAL
}

\author{
Florencia Barrios Tirado \\ SANTIAGO: UNIVERSIDAD DE CHILE, 1983 \\ (UNIVERSITARIA).
}

(Fragmento) 


\section{CAPÍTULO V: EL LICEO EXPERIMENTAL MANUEL DE SALAS: UN HITO EN LA EDUCACIÓN CHILENA}

Al resumir y completar el aporte del Liceo Experimental "Manuel de Salas" a la Educación Chilena, ha sido necesario insistir en algunos de los temas tratados con anterioridad, a fin de destacar la importancia y validez de sus planteamientos que son de muy fácil aplicación en cualquier ambiente educacional moderno.

Al citar en el capítulo primero el Decreto de creación del Liceo "Manuel de Salas", se vio que en sus considerados se decía, sobre su misión, lo siguiente: "Ensayar reformas de organización didáctica, nuevos métodos de enseñanza y diferentes agrupaciones de materias en los programas" y además, que "tales innovaciones ensayadas y vigiladas, en un establecimiento adecuado, permitirán dar base sólida a las reformas que se intentan en la Educación Secundaria General” (17).

En los capítulos anteriores se mostró el nacimiento del Liceo, sus primeros pasos, su niñez, su adolescencia, su juventud y su madurez. En todos esos periodos, hubo etapas de crisis, de vicisitudes, de inquietudes, de incertidumbre, de inestabilidad; pero siempre en su devenir histórico hubo creación, entrega, mística y sobre todo, un trabajo anónimo de muchos que permitió que se cumpliera a cabalidad la misión encomendada.

Después de recorrer la larga ruta de sus cincuenta años, puede afirmarse que en ningún momento esa comunidad de directivos-docentes, docentes, paradocentes, persona y administrativo y personal de servicio, dejó de cumplir con su misión y además, siempre estuvo en su espíritu en su quehacer diario la formación integral de sus educandos y gracias a todo ello, ha habido un aporte enriquecedor a la Educación Secundaria Chilena.

Se puede afirmar que la emisión fue cumplida; pues esa comunidad llegó a constituirse en una familia de profesores, paradocentes, tecnicodocentes, administrativos, padres, apoderados, amigos del Liceo, auxiliares y directivos, todos los cuales tienen un hijo común y multitudinario, el alumno, que se formó bajo su alero.

El optimismo de lo afirmado en el párrafo anterior, no es producto de un subjetivismo sentimental, pues allí está la obra de la semilla pródiga que ha caído en el surco fructífero de la Patria. 
En ninguna de las reformas que se han realizado en la Educación Secundaria Chilena desde la década del 30 a la década del 70, ha estado ajeno el acervo pedagógico del Liceo Experimental "Manuel de Salas",

Podrá parecer pretensiosa la afirmación anterior, y es por eso que en este capítulo se mostrará el aporte que ha entregado el Liceo de Educación Chilena, mientras estuvo bajo la tuición de la Universidad de Chile.

Aporte que no habría sido posible sin la grandeza de la Universidad de Chile, institución venerable que no solo permitió las experiencias pedagógicas, sino que las incentivó, creando la atmósfera propicia para que se realizaran y además, con esa generosidad que solo tienen los Institutos de renombre internacional, dio lugar a que se expandieran, entregándolas a la Comunidad Nacional.

Se puede afirmar que el aporte de la Universidad de Chile a la Educación Secundaria ha sido el de mayor magnitud que ha recibido el Ministerio de Educación desde la instauración de la República, que provino de la propia realidad nacional y ello permitió fortalecer en los educandos el amor a su país, a su comunidad y a su familia, creando en ellos una actitud de servicio y de entrega a la misión que debían cumplir en su vida productiva, la que se enraíza en el Liceo chileno, enriquecido gracias a la experimentación del Liceo "Manuel de Salas".

Para cumplir su misión, el Liceo Experimental "Manuel de Salas", creó su propia filosofía educacional basada en la adhesión a los principios que garantizan la mejor convivencia dentro de la sociedad democrática, principios que permiten al individuo, junto con el ejercicio de las libertades y derechos, propender al desarrollo de su personalidad, para cumplir con eficiencia sus responsabilidades frente a la colectividad nacional.

Se consideró la educación como un proceso que capacita al individuo para adaptarse al medio en que vive, interpretarlo y mejorarlo; proceso educativo que en el Liceo Experimental "Manuel de Salas" se orientó de acuerdo a las finalidades de proporcionar al individuo las más amplias oportunidades para el desenvolvimiento de sus capacidades, atendiendo a sus diferencias especiales, sus intereses y aptitudes y además, se preocupó de preparar al tipo de ciudadano que nuestra realidad nacional reclama, a través de experiencias y actividades que le han permitido comprender e interpretar sus problemas sobresalientes, de modo que se han desarrollado en él aquellos valores permanentes, actitudes y habilidad que lo han capacitado para actuar eficientemente en el medio social y han contribuido a su mejoramiento y al engrandecimiento de su Nación.

De acuerdo a su filosofía, a la realización de sus finalidades y a la misión encomendada, la entrega ha sido la siguiente: 


\section{NUEVA ORGANIZACIÓN GENERAL DE ESTUDIOS}

Ensayó con éxito diferentes planes de estudio y el Liceo Chileno ha tomado y está tomando algunos de los tres órdenes de actividades en que el Liceo "Manuel de Salas" organizó los estudios, de acuerdo al siguiente esquema:

a) Un Plan Común mediante el cual el Liceo "Manuel de Salas" comparte la preocupación esencial de la enseñanza secundaria en el sentido de seleccionar un conjunto de conocimientos y actividades que contribuyan a explorar y satisfacer las necesidades e intereses comunes de los adolescentes. A través de este Plan Común se arbitran, en especial, los recursos tendientes a promover el desarrollo de la personalidad de los alumnos ejercitándolos en el pensamiento reflexivo y en la adquisición de una cultura general que los capacite para la resolución y la acción inteligente frente al mundo natural y cultural en que se desenvuelven.

b) Un Plan Variable en el que, sobre la base de una selección de experiencias, actividades e informaciones, se tiende a que el educando descubra su vocación o profundice y sistematice conocimientos y técnicas en relación con sus especiales intereses, capacidades y aptitudes, $y$

c) Cursos Diferenciados en el $3^{\circ}$ y $4^{\circ}$ año de humanidades, en los que acentúan los conocimientos relativos a tres áreas de la cultura: Letra, Biología y Matemática, elegidas por los estudiantes.

El Plan Variable -que se extiende de $1^{\circ}$ a $2^{\circ}$ año de humanidades- constituye la etapa en la cual el alumno habrá de decidir su elección con respecto a las tres áreas de los Cursos Diferenciados.

Tanto a través del Plan Común como del Plan Variable y de los Cursos Diferenciados, el Liceo "Manuel de Salas" acentúa el principio de que las actividades y conocimientos que forman parte del contenido de las diversas asignaturas o materias que se ofrecen al alumno no solo deben ser seleccionados debidamente, sino que, además, deben ser graduados de acuerdo, por una parte, con el desarrollo biosociológico del estudiante y por otra, con las exigencias que la vida nacional le plantea dentro del proceso total de la cultura.

Estos Planes fueron tomados en plenitud en los Liceos Experimentales que creó el ministerio de Educación el año 1946 y solo parcialmente por los liceos tradicionales, posteriormente, al ser absorbidos estos Liceos Experimentales por el Liceo Común. Estos últimos continuaron recogiendo experiencias del Liceo "Manuel de Salas" y es así como en los últimos Planes y Programas para la Educación Media Humanística-Científica aprobado por el MINEDUC en diciembre pasado, integraron a ellos, nuevas experiencias de éste.

De esta misma experimentación provienen los planes variables; los cursos flexibles; los grupos flexibles de recuperación; las tutorías pedagógicas; los centros 
de estudio; las academias científicas, artísticas y culturales y los clubes; todos los cuales fueron ensayando y evaluando en décadas pasadas y que, ahora son ofrecidos a los educandos de algunos colegios particulares como una gran novedad pedagógica.

\section{ORGANIZACIÓN DE SUS ACTIVIDADES SOBRE LA BASE DE LA COEDUCACIÓN.}

Fue el primer Liceo de la República que implantó la coeducación, pues al implantar tal régimen en todas sus actividades, aseguraba una efectiva convivencia democrática, la que no podía establecerse basada en discriminaciones de sexo.

Además, en ese medio todos sus integrantes compartían solidariamente de acuerdo a sus aptitudes e intereses, sus ideales y responsabilidades, sin distinción de sexos, asegurando a cada uno de ellos iguales posibilidades y derechos.

En los educandos se fortalecieron sus actividades sociales y se les adentró en el respeto y la confraternidad mutua entre hombre y mujer, en un periodo de su vida tan rico como la adolescencia, en el que se sufren los problemas más serios, intelectuales, emocionales y sociales, ya que los adolescentes viven en plena búsqueda de valores para formar su propia filosofía, la que les sirve de motor espiritual para su avance hacia el futuro.

La coeducación no existía en el medio chileno de la década del 30 y solo 15 años después de su implantación se extendió a algunos Liceos de la República. Lo que ahora es normal, es esa lejana época, cincuenta años atrás, era escándalo. Sin embargo, la diafanidad del alma adolescente de los alumnos y de las alumnas del "Manuel de Salas", y la mística de sus profesores y profesaros, permitió que una experiencia tan vital y enriquecedora no se malograra. Ahora se ha casi generalizado en todos los establecimientos secundarios de la Nación.

\section{ATENCIÓN A LAS DIFERENCIAS INDIVIDUALES}

Para el Liceo, el alumno dejó de ser considerado como un elemento anónimo en el grupo-curso, transformándose en persona con una individualidad, diferente y única de cualquier otra, y por ello, entendió a sus diferencias individuales tanto en su aspecto físico, como mental y social.

Además, se consideró que tales diferencias debían ser atendidas por especialistas y, para ello, creó servicios especiales de orientación, de servicio médico y de servicio social.
a) Servicio de Orientación 
A través del Servicio de Orientación se preocupó de atender todos los aspectos de la personalidad del educando, centralizándolo en la Orientación personal, Orientación vocacional y educacional y Orientación social.

En un principio, cuando no existía la profesión de Orientador, colocó a cargo de las mencionadas Secciones al profesor-consejero, quien había recibido una formación de postgrado que lo capacitaba para cumplir tales funciones.

En el año 1946, recién se incorporó la orientación en los liceos experimentales del Ministerio de Educación y posteriormente, en la década del 50, cuando la Universidad de Chile ofreció la carrera de Orientador, tal Servicio empezó a crearse en los Liceos de la República a lo largo de los decenios 60 y de parte de 70 .

Es decir, un Servicio de orientación implantado en el "Manuel de Salas" en la década del 30, dio origen a la profesión de Orientador en los años 50 y a la generalización posterior de tal atención a los educandos del nivel medio a partir del 70.

Además, al finalizar la década del 50, el Liceo puso en función, para fortalecer la Orientación, a los Psicólogos Educacionales, con el objeto de tratar aquellos alumnos que presentaban desequilibrios psicológicos que dificultaban su adaptación a los medios sociales y a su normal rendimiento. La atención del Psicólogo Educacional, en el medio estudiantil secundario del país, solo se ha implantado en una mínima parte, en algunos establecimientos educacionales, a pesar de la importancia que tal disciplina tiene para resolver algunas dificultades propias del educando en su edad adolescente.

b) Servicio Médico

El Liceo implantó el primer Servicio Médico dinámico del país el cual, aun hoy, no ha sido superado por ningún colegio de la República.

Este Servicio Médico no solo realiza una atención preventiva, sino que hace un seguimiento al tratamiento de las deficiencias patológicas de aquellos alumnos que presentan problemas en su salud; también hace algunas pequeñas intervenciones quirúrgicas y realiza visitas domiciliarias cuando se le requiere. Su equipamiento e infraestructura es tal que cuenta con una moderna ambulancia.

Junto al Servicio Médico está el Servicio Dental, el que realiza revisiones periódicas al alumnado y ejecuta tratamientos dentales.

c) Servicio Social

Así como el Liceo fue pionero en el Servicio Médico-Dental; lo es también el Servicio Social.

Este Servicio se preocupa de resolver todos aquellos problemas socioeconómicos que tienen algunos alumnos y ello ha permitido que actualmente numerosos profesionales de renombre hayan alcanzado el nivel que ocupan gracias a la ayuda silenciosa del Servicio Social y más que eso, algunas familias que se estaban 
desintegrando con el consiguiente trauma para el educando, superaron la crisis con la intervención de la asistente social.

También es función de este Servicio extender la ayuda a la comunidad y gracias a tal encauzamiento se han construido escuelas, equipado colegios, fomentado el deporte en áreas marginales, se han construido caminos, levantando viviendas y tantas otras obras materiales que sería interminable su enumeración. Sin embargo, nada habría sido posible sin la valiosa colaboración de los profesores, alumnos, padres, apoderados y amigos del Liceo.

Así como se dijo que el Liceo Experimental "Manuel de Salas” creó el primer Servicio de Orientación y el primer Servicio Médico-Dental Dinámico, puede decirse que creó también el primer Servicio Asistencial activo.

El Liceo Experimental "Manuel de Salas" ha considerado y considera que tanto la salud del educando, como el equilibrio social y familiar, son los elementos esenciales para que el alumnado alcance, a través de sus estudios y la adecuada orientación vocacional, la plenitud de su formación. Es por eso que su gran mérito y originalidad ha sido la incorporación a los Consejos Técnicos, de Diagnóstico y de Evaluación de aquellos profesionales a cargo de los Servicios de Orientación, Médico-Dental y Social.

\section{ORGANIZACIÓN DE LA VIDA ESCOLAR}

EL Liceo organizó su vida escolar de acuerdo a la experiencia y práctica y después de ensayar diversas formas de acuerdo a la hipótesis propuesta, creo la más adecuada a la realidad educacional Nacional y a su propia realidad.

El Liceo, en su organización, aseguró la participación de cada uno de sus miembros, incluyendo a los directivos-docentes, personal docente, personal administrativo, personal técnico y personal médico y paramédico, pues estimaba que todos ellos jugaban un rol importante en el planteamiento y orientación de la vida escolar, en el proceso de su desarrollo y evaluación de sus resultados, ya que todos, por ser una empresa colectiva debían mantener una permanente y recíproca cooperación.

\section{a) Departamento Técnico}

Creó organismos que aun no han sido incorporados a otros Liceos del país, en el caso del Departamento Técnico, a cargo de un Asesor Técnico y del cual dependían las Secciones de Profesores de Asignaturas, Sección de Profesores Guías, Sección Instituciones Estudiantiles, Sección Profesores Jefes y Sección Orientación Educacional y Vocacional. 
El Liceo fue el primero que creó la Sección de Profesores de Asignatura del país; el primero en crear la Sección de Profesores Guías, encargados del planear, organizar y supervisar la práctica pedagógica de los alumnos que estudiaban pedagogía, tal Sección no ha sido formada en los otros Liceos y en cambio, han continuado con la práctica aislada de tener profesores guías sin ninguna coordinación dentro del establecimiento, fue el primero en crear los Gobiernos Estudiantiles y por ende, la Sección Instituciones Estudiantiles.

Fue pionero en la creación de la Sección Profesores Jefes, pues se hacía necesaria la planificación conjunta del trabajo pedagógico que realizaba con los alumnos, se desarrollaban Unidades especiales y por último, se creó la Sección de Orientación Educacional y Vocacional.

\section{b) Consejos Especiales}

El consejo general de profesores nació en el liceo chileno y en él se trataba bimestralmente la situación de los educandos. Estos consejos tenían una duración máxima de dos horas, lo cual hacía imposible un análisis detallado de los casos individuales. Además. Durante el desarrollo del periodo escolar, los profesores no tenían ninguna relación, ya que se tratara de profesores-jefes, profesores de asignaturas afines o profesores de un determinado curso.

El Liceo "Manuel de Salas", desde sus inicios, creó una serie de Consejos Especiales tendientes a mejorar el trabajo pedagógico sobre todo a respetar las características individuales de los educandos y a tratar y evaluar en profundidad la vida y resultados obtenidos por los estudiantes.

$\mathrm{Al}$ respecto, el Liceo aportó a la Educación Secundaria, los siguientes consejos:

1. Consejo Técnico, encargado de estudiar y de resolver con éxito los problemas técnico-educacionales que presentan el planteamiento y desarrollo de la experimentación en el establecimiento. Este consejo no ha sido incorporado al Liceo tradicional.

2. Consejo de Departamentos Especiales, como el de Bienestar, (Médico-Dental, Asistencia Social); tal consejo no ha sido incorporado aun al Liceo tradicional.

3. Consejo de Evaluación por Cursos, el cual ha sido incorporado desde la década del 50 al Liceo chileno.

4. Consejo de Profesores Jefes, ha sido incorporado al Liceo tradicional en forma parcial desde la década del 70 en adelante.

5. Consejo de Asesores de Instituciones Estudiantiles, tal consejo no ha sido incorporado aun al Liceo chileno.

6. Sobre los Consejos de Profesores por Asignatura, se hablará en el punto siguiente cuando se traten las Secciones y Departamentos por asignaturas.

7. Por último, sobre el Consejo General de Profesores, aun cuando provenía del Liceo Común, en el Liceo "Manuel de Salas" adquirió una nueva modalidad no solo por 
las materias que se trataban (nuevas modalidades docentes; estímulos a los alumnos y otras personas vinculadas al Liceo; distribución de los premios a los alumnos que egresaban del Establecimiento; reconocimiento general a los profesores meritorios; concurrencia de altas personalidades del campo de la Educación y de la Universidad de Chile; aprobación de jornadas de estudio; planeamiento conjunto de actividades comunes tanto en el plano docente, paradocentes, académico, paraacadémico, social, ayuda solidaria, etc.); sino que también porque incorporo a él a otros profesionales del Liceo; psicólogas, médicos, asistentes sociales, bibliotecarias y orientadores.

Además de las modalidades indicadas en el párrafo anterior y que le han dado una originalidad muy propia, se agrega el hecho de que dentro de sus atribuciones está el conocer el "Plan General de Trabajo del Año Escolar" que le presenta la Dirección del Liceo y puede sugerir las medidas tendientes a su mejor realización y por último, conocer la Memoria Anual del Establecimiento, que la Dirección debe entregar a las Autoridades Superiores de la Universidad.

Un Consejo General de Profesores con tales modalidades, aun no existe en la gran mayoría de los establecimientos del país.

c) Secciones por Asignar

Otro aporte del Liceo fue la creación de las Secciones por Asignaturas. La misión de sus integrantes era preparar el material didáctico uniforme para el desarrollo de los programas de estudio, como asimismo, los tipos de pruebas y ejercicios que servían como pautas objetivas de control y evaluación del rendimiento de los alumnos.

También preparaban guías de estudio que se utilizaban en reemplazo de los textos tradicionales, que por su rigidez no eran los auxiliares más adecuados para el proceso de enseñanza-aprendizaje.

Al igual, realizaba un estudio crítico de los resultados de todas las pruebas de control y de los criterios empelados en la evaluación.

Se preocupaba de redactar guías bibliográficas de sus respectivas asignaturas a fin de que fueran adquiridos posteriormente, pasando a constituir patrocinio de la Biblioteca del Liceo.

Propendía a la publicación de materiales didácticos o de divulgación relativos a sus asignaturas.

Las secciones de asignaturas han sido incorporadas al Liceo chileno.

d) Escuela Primara Anexa

El Liceo incorporó a su ámbito una Escuela Primera a la que dio una organización semejante de la del Lico.

Contaban con un Jefe de Departamento que cumplía funciones administrativas y técnicas y por estas últimas, era integrante del consejo técnico del Liceo. 
La organización de la Escuela Anexa se basaba en dos organismos colegiados: Consejo General de Profesores y Consejo de Grado o Nivel. Además, tenía tres Secciones especiales: de Profesores Jefes, de Evaluación y de Psicología Educacional.

Así como preocupaba al Liceo la formación integral del adolecente, le preocupaba también la formación integral del niño y para lograrlo, creó las secciones anteriormente nombradas, las cuales solo se han incorporado parcialmente, en la década pasada, en algunos Colegios Básicos.

\section{NUEVOS ASPECTOS METODOLÓGICOS.}

El Liceo considera que las actividades, experiencias y conocimientos que se entregan al alumno deben ser seleccionados y graduados de acuerdo al nivel alcanzado en su desarrollo biológico, intelectual y emocional y además, acorde también con el proceso total de la cultura y con las exigencias que el bien nacional plantea a la educación.

Esta selección y graduación de materias de acuerdo a los postulados planteados, fueron únicos en su época y el esfuerzo más serio para emularlos solo se ha dado en la presente década.

Junto a los contenidos están los métodos de trabajo, pues el Liceo reconoce que los procedimientos y técnicas que se emplean en la realización de sus programas, deben ajustarse a las modernas técnicas del aprendizaje, es decir, aprender con su intelecto, sentirlo y en sus sentimientos y aplicarlos en su acción. Al alumno debe enseñársele a pensar, a razonar, a discernir, a valorar su inteligencia, a respetar sus sentimientos y a fomentar su desarrollo corporal.

El Liceo Experimental "Manuel de Salas" propugna un completo desarrollo de la personalidad de los alumnos mediante el descubrimiento de sus propias potencialidades y para ello, fomenta su permanente participación a través de clases activas y previamente motivadas y no solo eso, sino que también participando en actividades paraacadémicas y además, tomando responsabilidades frente al grupocurso, a la comunidad liceana, a su familia y a la colectividad en la cual vive.

En síntesis, ha transformado la actividad escolar en un propósito que tiene significado y valor para el individuo y grupo que la realiza; pues mientras mayor significado vital se logre y mayor sea la conexión que sobre esa activad se tenga, más firme, duradero, efectivo y productivo será el aprendizaje adquirido.

El Liceo fue uno de los primeros en emplear los siguientes métodos: recitación grupal, método de proyectos, método de problemas, lectura dirigida, investigación dirigida, método expositivo, métodos de unidades de trabajo, actividades dirigidas y tantos otros que sería larga su enumeración. 
Todos estos nuevos aspectos metodológicos, se vienen aplicando y ensayando, al igual que otros, desde la década del 30 en el "Manuel de Salas". Sin embargo, solo en el año 1946 empezaron a aplicarse algunos de los Liceos Experimentales que creó el MINEDUC.

\section{MÉTODOS MODERNOS DE ENSEÑANZA.}

El Liceo se ha preocupado de incorporar a su medio los más modernos métodos de enseñanza. Destacan entre otros los siguientes:

a) Laboratorio de Lengua. Fue el primer colegio que creó un laboratorio de lenguas con casetas individuales para un curso completo, en donde el alumno puede grabar su pronunciaron y corregirla las veces que se quiera, al poder compararla con la pronunciación perfecta que puede escuchar, también, las veces que desea.

Este valioso Laboratorio de Lenguas, aun está lejos de ser incorporado al Liceo chileno, en circunstancias de que tal modalidad de trabajo es ajena a los otros centros de enseñanza secundaria de otros medios más tecnificados.

b) Televisión Educativa. En el año 1960 y hasta el año 1963, el Liceo realizó el primer "Proyecto Experimental de Televisión Educativa" desarrollado en el país.

En una época en que la televisión era prácticamente desconocida en nuestro medio, esta comunidad emprendió la importancia que tal recurso podía tener para los fines educativos.

Esa experiencia que tuvo en el año 1961 un carácter eminentemente experimental, gracias al cumplimiento de los objetivos propuestos, se amplió en el año 1962 a tres mil alumnos de los Liceos Gabriela Mistral, Valentín Letelier, Aplicación, de Niñas $n^{\circ} 4$ y Barros Borgoño, y es así como semanalmente se beneficiaron con los programas de televisión educativa, los cuales complementaron y enriquecieron sus actividades escolares.

Como los resultados de esta segunda etapa fueron óptimos, al año siguiente se realizó un plan más amplio que incluyó, además de lo ya realizado un Programa de Televisión para todo público y un Seminario para preparar a los profesores básicos y medios en el adecuado uso de la televisión para fines educacionales.

La experiencia fue enriquecedora y sirvió de base para otros programas de televisión educativa que surgieron, como una gran novedad, en la década del 70. Sin embargo, la experiencia lograda está aún lejos de incorporarse, a través de circuitos cerrados de televisión, en los medios educacionales del país, lo que sería una valiosa ayuda al proceso enseñanza-aprendizaje.

c) Laboratorio y Talleres. El liceo creó laboratorios de Física, Biología, Química y Talleres de Técnicas Especiales, de Artes Plásticas y de Música. 
Podría pensarse que tales medios existen en otros liceos del país, pero en el Liceo "Manuel de Salas", por su propio carácter experimental, se crearon tales medios necesarios para la investigación y el desarrollo de las Artes, derogándolos de los elementos que caracterizan un centro científico y de aquellos que utilizan los artistas en sus academias y los técnicos en sus talleres.

Sus laboratorios y talleres son centros dinámicos que han gozado de la más amplia libertad para desarrollar en los educandos su espíritu científico, o bien, su espíritu artístico o por último, su aptitud técnica; todo de acuerdo a sus propias condiciones o inclinaciones.

De ahí la originalidad de sus laboratorios y talleres, ya que estos últimos, por tener el mismo nivel de los primeros, presentan en su conjunto valiosos medios modernos de enseñanza, que los convierten en únicos entre sus congéneres de otros Liceos del país.

\section{NUEVO MATERIAL DIDÁCTICO.}

En el Liceo, el profesor elabora el material didáctico necesario para satisfacer tanto sus propias aspiraciones de carácter profesional como las especiales características individuales y de los grupos-cursos para los cuales están destinados.

El trabajo en equipo ha permitido una permanente realización de materiales didácticos originales para cada una de las asignaturas al igual que para sus actividades complementarias.

a) Guías de Estudio y Ejercicios

La confección de guías de estudio y guías de ejercicios orientadas por las respectivas Secciones de Asignaturas, convierten al profesor en un elemento dinámico del proceso educativo, eliminándose así la posibilidad de que se conviertan en elementos pasivos, apoyados en un material ajeno a las circunstancias especiales que se presentan, necesariamente, en el proceso del aprendizaje.

b) Diarios Murales y Exposiciones

A estos aportes del Liceo "Manuel de Salas" se agregan otros complementos como los Diarios Murales por Cursos, Diarios murales generales del colegio, exposiciones científicas, artísticas y culturas, auxiliares valiosos para reforzar el proceso enseñanza-aprendizaje y también a aquellos valores intrínsecos a la Nacionalidad, a la Historia, al medio natural y cultural.

Estas modalidades tan originales y tan útiles para el educando, han sido utilizadas solo esporádicamente en el medio educacional chileno, a partir de la década del 40. 


\section{c) Publicaciones*}

La creación de este nuevo material didáctico, se entregó a los profesores de la República a través de numerosas impresiones y para ello, el Liceo creó su propia Sección Publicaciones.

Además, como incentivo para la publicación de trabajos de creación los profesores, la sección publicaciones ofrecía numerosos estímulos.

Para los efectos de determinar acerca de la publicación de escritos originales, intervenía una Comisión integradora por la Dirección del Liceo, el Jefe de la Sección de Publicaciones y un profesor designado especialmente en cada caso.

La sección publicaciones desarrolló una extensa labor que se resume como la siguiente:

1. 1934 Primera Memoria Anual del Liceo.

2. 1934 Información presentada a la Exposición Interamericana de Educación, en la que se suministran noticias varias sobre el funcionamiento del Liceo "Manuel de Salas".

3. 1940 Folleto intitulado "Bernardo O’Higgins, Padre de la Patria", escrito a solitud del Ministerio de Educación. Se refiere al periodo de la historia de Chile que comprende la vida de este prócer chileno.

4. 1941 "Programa de Chilenidad". Plan presentado por el Liceo al Ministerio de Educación en el que se expone una serie de sugestiones para ensayar pedagógicamente una obra de chilenidad, sobre la base de las distintas materias que componen los programas de enseñanza secundaria. En este trabajo colaboró la totalidad de los profesores de este Liceo.

5. 1941 "Ideario de Manuel de Salas", publicado bajo los auspicios del Ministerio de Educación, como homenaje en el primer centenario del fallecimiento de don Manuel de Salas.

6. 1943 "Algunas Consideraciones sobre la Enseñanza Moderna de las Ciencias Naturales" del profesor don Natalio Clavic Richardson.

7. 1943 Boletín n 1 , destinado a divulgar algunos de los resultados de mayor interés en la labor de orientación vocacional realizada hasta entonces en el Liceo.

8. 1944 Boletín $n^{\circ} 2$, dedicado en su totalidad al estudio de las actividades extra programáticas.

9. 1944 "Guías de Estudio y Ejercicio correspondientes al programa de Historia y Geografía", para cuarto año de humanidades. Trabajo de la profesora, doña Olga Poblete de Espinoza.

10. 1944 “Guías de Estudio y Ejercicios para la Enseñanza Correlacionada de las Ciencias Naturales, correspondientes al programa de segundo año de humanidades, escrito por doña Florencia Barrios Tirado. 
11. 1945 Boletín $n^{\circ} 3$, en que se divulga la filosofía educación del Liceo y la organización de su vida escolar.

12. 1945 "Declaración de los principios educacionales del Liceo Manuel de Salas.

13. 1945 "Guía de Matemáticas". Colaboración de las profesores señoritas María Lara Poblete y Zulema Valdivieso Chamberyron.

14. 1948 "Guía del Estudiante del Liceo Experimental Manuel de Salas". Contiene un conjunto de informaciones breves para orientar al alumno acerca de la especial modalidad de este Liceo y a facilitar, por consiguiente, su adaptación.

15. 1951 "Historia de Europa Moderna". Unidad sobre el Renacimiento y la Reforma, para quinto año de humanidades, elaborado por los profesores de la sección de estudios sociales del liceo.

16. 1951 "Ideas para una Teoría de la Personalidad". Trabajo de doña Florencia Barrios Torado, dirigido a dilucidar el problema del desarrollo de la personalidad y los diversos factores que en él intervienen.

17. 1951 "Material de consulta de Geografía de Chile", para el Primer Ciclo de Humanidades, confeccionado por la Sección de Estudios Sociales del Liceo.

18. 1951 "Material de consulta sobre "maquinismo", para el primer ciclo de humanidades, preparado y redactado por la sección de estudios sociales del liceo.

19. 1951 "Instrucciones Generales acerca de los Actos Académicos", para el primer año de humanidades. Constituye breves indicaciones redactadas por la sección de castellano del liceo, para dirigir a los alumnos en la exposición de temas de obligación escolar.

20. 1951 "Poesía y Recitación", para el primer año de humanidades, obra de la Sección de Castellano. Es un trabajo en que se dan nociones sencillas relacionadas con el título. Se incluyen, además, algunos poemas escogidos para ejercicios de recitación.

21. 1952 "Historia del Teatro en Chile", por el profesor de Castellano del Liceo, señor Zlatko Brncic. Y contiene la historia de la actividad teatral chilena durante la conquista y la colonia. Esta publicación fue financiada por la Universidad de Chile.

22. 1953 "Cuadernos de Estudios Social", para primer año de humanidades. Trabajo realizado en conjunto por la sección de Estudios sociales.

23. 1953 "Cuadernos de estudios sociales" unidad de trabajo complementaria del anterior.

24. 1953 "Relaciones entre la Educación Secundaria y la Universidad". Ponencia presentada por el Liceo al Segundo Congreso y Primera Asamblea de la Unión de Universidades Latinoamericanas.

25. 1953 "Documentos para el estudio de la historia de la antigüedad" de la profesora doña Olga Poblete de Espinoza, con prólogo del rector de la universidad, don Juan Gómez Millas. 
26. 1953 “Apuntes para una biografía del liceo Manuel de Salas". Es una historia breve del liceo escrita por el profesor Alberto Arenas.

27. 1953 "Bases Psicológicas para la Educación de la Adolescencia", del profesor don Oscar Ahumada Bustos. Es un ensayo de información y ayuda para padres y apoderados en la tarea de la educación del adolescente.

28. 1953 "Agenda Escolar" para los alumnos del Liceo "Manuel de Salas. Constituye uno de los medios de que se vale el liceo para ponerse en contacto con el hogar de sus alumnos. Contiene un conjunto de recomendaciones para los alumnos, el reglamento de calificaciones y promociones, el horario de clases, el calendario de tareas y pruebas, páginas para el control de biblioteca, de atrasos e inasistencias; para consignar los cargos que desempeñe el estudiante en Consejos de Curso e Instituciones Estudiantiles; para conminaciones del hogar al Liceo y viceversa, etc.

29. 1954 "Naturaleza y funcionamiento de los consejos de curso" folleto informativo sobre el tema, escrito sobre la base de las experiencias del profesorado del liceo sobre esta actividad escolar.

30. 1954 "Cuaderno de lógica y ejercicios" para el estudio de la materia del programa de quinto año de humanidades. Trabajo de la profesora doña Olga Poblete de Espinoza.

32. 1955 "Cuaderno de estudios sociales", para el segundo año de humanidades, con ilustraciones y materiales de consulta, de la profesora doña Olga Poblete de Espinoza.

33. 1955 "Cuaderno de estudios sociales" para el segundo año de humanidades, con ilustraciones y materiales de consulta, de la profesora doña Olga Poblete de Espinoza.

34. 1955 "Cuaderno de Trabajos de estudios sociales" para segundo año de humanidades. Ejercicios redactados por la profesora doña Olga Poblete de Espinoza.

35. 1956 "Cuadernos de trabajo de francés" para primer año de humanidades. Trabajo de la profesora señora Ana Fragnaud de Martínez.

36. 1956 Boletín no 4 que contiene el plan de experimentación educacional del liceo.

37. 1957 "Metodología de la enseñanza de las ciencias biológicas", del profesor señor Natalio Glavic Richardson.

38. 1958 "Cuaderno de francés" para segundo año de humanidades, de la profesora señora Ana Fragnaud de Martínez.

39. 1958 "Cuadernos de francés" para tercer año de humanidades, de la profesora señora Ana Fragnaud de Martínez.

40. 1958 "Agenda escolar" para los alumnos. $2^{\circ}$ edición.

41.1958 "Cuaderno de francés" para primer año de humanidades, de la profesora señora Ana Fragnaud de Martínez. $2^{\circ}$ edición. 
42. 1959 "Cuadernos de francés" para cuarto año de humanidades, de la profesora señora Ana Fragnaud de Martínez.

43. 1959 "Psicología Fundamental" del profesor don Oscar Ahumada Bustos.

44. 1960 "Cuadernos de francés" para quinto año de humanidades, de la profesora señora Ana Fragnaud de Martínez.

45. 1960 Boletín $\mathrm{n}^{\circ}$ 5. Presenta un balance del plan de experimentación educacional del liceo.

46. 1960 Boletín sobre 20 años de labor de la CORFO.

47. 1961 "Cuaderno de francés" para segundo año de humanidades, de la profesora señora Ana Fragnaud de Martínez. $2^{\circ}$ edición.

48. 1961 "Unidades de Estudio de historia de las Artes plásticas" de la profesora doña Amanda Flores de Preotti. Cuatro unidades: 1. Renacimiento en Italia y su expansión en Europa. 2. Arte cristiano y arte árabe. 3. Estilo barroco en Italia. Expansión en Europa Estilo rococó. 4. Manifestaciones plásticas de los pueblos orientales. 5. Resurgimiento clásico. Época contemporánea.

50. 1962 "Agenda escolar", $3^{\circ}$ edición.

51.1962 "Cuaderno de logia con ejercicios y nociones de teoría de conocimiento", del profesor don Oscar Ahumada Bustos. $2^{\circ}$ edición.

52. 1962 "Los alimentos y problemas de la alimentación", del profesor Natalio Glavic Richardson.

53. 1962 "Psicología fundamental", del profesor don Oscar Ahumada Bustos. $2^{\circ}$ edition.

54. 1962 "Selection readin pieces for senior stundets" (Booklet numer one and Booklet numer two) por las profesoras señora Raquel Varas Latorre y Olga Lopenhandia de Meza.

55. 1962 "Bajo el Cielo de Chile". Dos volúmenes: "El paisaje de mi tierra" y "mi patria", del profesor de Castellano del Liceo don César Reyes Beas.

56. 1965 "Agenda escolar" 4 edición.

57. 1965 "Piscología Fundamental", del profesor don Oscar Ahumada Bustos. $3^{\circ}$ edición.

58. 1965 "El funcionamiento de nuestro régimen democrático". Material de educación cívica, del profesor del ramo señor Sergio de los Reyes Ibarra.

59.1966 “Carpeta Docente del profesor”. Recopilación de Decretos, reglamentos y circulares.

60. 1968 "Fundación Manuel de Salas". Anuario del año 1968.

61. 1969 "Agenda escolar" 5 edición.

62. 1969 "Tres años de Televisión educativa". Ediciones de la Universidad de Chile. Profesora a cargo del proyecto doña Viola Soto Guzmán. 
Todas estas publicaciones forman parte de los Modernos Métodos de Enseñanza que el Liceo Experimental "Manuel de Salas” ha aportado a la educación secundaria.

\section{SISTEMAS ESPECIALES DE EVALUACIÓN.}

El liceo adoptó los más modernos sistemas de evaluación, midiendo el conocimiento adquirido a través de pruebas objetivas preparadas por el personal docente. Además, se preocupó de evaluar las nuevas formas de conducta y los ideales que los educandos van adquiriendo a lo largo de su vida escolar.

El liceo consideró que la apreciación del trabajo escolar, no podía limitarse solo a la adquisición de las materias de estudio, pues ello tergiversa de manera negativa, el verdadero significado del sistema educativo, lo importante es medir el proceso de transformaciones en la conducta del educando en el sentido de reaccionar de manera inteligente y favorable en el medio en el que le corresponde actuar.

En síntesis, es necesario evaluar en el alumno su crecimiento en cuanto a los conceptos que incorpora a su acervo cultural como también las formas más sutiles del desarrollo de su personalidad.

Para lograr lo anterior, el liceo creó nuevos instrumentos de apreciación y cuyas formas varían de acuerdo con los rasgos que se intentan evaluar.

Las pruebas basadas en el desarrollo de temas, han sido substituidas por variadas formas que ofrecen los exámenes de nuevo tipo y por otras técnicas de evaluación de carácter objetivo, de control y diagnóstico del aprendizaje, mediciones mentales, exploración de aptitudes e intereses, estimación de los cambios operados en el desarrollo de la personalidad, aplicación de cuestionarios, entrevistas y observaciones, análisis de anécdotas y de autobiografías, etc.

En cuanto a las actitudes sociales, se puede decir que éstas son evaluadas por todos los profesores en forma permanente a través de las actividades que realizan los alumnos del liceo "Manuel de Salas", calificándose así, aquellos aspectos de su personalidad que tienen especial importancia en el fortalecimiento de su quehacer social.

Estas actitudes sociales se refieren a los siguientes aspectos:

a) Cooperación en las actividades de grupo.

b) Responsabilidad.

c) Honradez en la vida escolar.

d) Iniciativa y capacidad de realización.

f) Cortesía. 
Estas actitudes sociales han sido consideradas fragmentarias en otros establecimientos del país. Sin embargo, la forma seguida para su evaluación, es totalmente distinta a la adoptada por el Liceo Manuel de Salas.

Para uniformar los criterios de evaluación, dictó una serie de normas especiales y además, creo una serie de pautas; de ellas se indican las siguientes:

Pautas y Escala para la Evaluación de las Actividades Sociales.

Pauta para discernir las distinciones a los alumnos de los sextos años de humanidades (actuales cuartos años de Enseñanza media).

Pautas para la evaluación de los alumnos en las actividades de las instituciones estudiantiles y en las labores individuales o colectivas en general.

Pauta para evaluar la participación del alumno en las labores del consejo de curso y del gobierno estudiantil.

Pauta para evaluar la participación del alumno en actividades paraacadémicas.

Pauta del profesor jefe para el consejo de evaluación semestral de profesores de curso.

Pauta de profesor jefe para presentar el consejo de evaluación anual de profesores de curso.

Estas pautas, de innegable utilidad, han sido incorporadas en forma parcial al liceo chileno.

\section{SISTEMA DE AUTODISCIPLINA}

La disciplina concierne a todo el personal del establecimiento, debido a como proponga no hay en el Liceo un personal especial de inspectores encargados de este importante aspecto de la vida escolar.

La autodisciplina requiere una convivencia efectiva de profesores y alumnos a través de las variadas actividades de los profesores; turnos, clases sistemáticas, instituciones estudiantiles, atención de practicantes en los cursos, visitas, excusiones, etc., tales actividades hacen posible una permanente observación y vigilancia del alumno y dan oportunidad para contribuir en la formación de hábitos y de un concepto claro de autodisciplina en los alumnos.

Esta modalidad practicada en el Liceo Manuel de Salas ha dado excelentes resultados que hacen recomendable su generalización en otros medios escolares.

\section{ACTIVIDADES EXTRA PROGRAMÁTICAS}

Otro aporte del Liceo Experimental Manuel de Salas, a la educación nacional, fueron las actividades extra programáticas que se han incentivado en su aplicación en otros establecimientos educacionales, desde la década del 70 en adelante. 
Dentro de estas actividades las contribuciones más importantes que destacan son; las semanas paraacadémicas, las competencias deportivas, las excusiones de estudio, las visitas a lugares de interés cultural y natural; la incentivación de la música a través de una escuela de música; del arte a través de la creación de una escuela de arte; y de la danza a través de una escuela de danza; la creación de talleres literarios y de teatro; la creación de conjuntos de música y de bailes folklóricos; la creación de academias científicas y otras que se enumeraran en los párrafos siguientes.

El Liceo ofrecía a los alumnos las siguientes organizaciones, entendiéndose que podrían funcionar otras que surgieran de las necesidades de la vida escolar o dejar de funcionar aquellas que durante el periodo de organización no representara para el alumnado intereses claramente definidos:

a) Academias: Literarias de estudios filosóficos y sociales, de ciencias naturales, de matemáticas, de artes plásticas, de artes musicales, de artes aplicadas.

b) Centros: Dramáticos de cooperación social, deportivo (constituido sobre la base de representantes de los cursos), de Publicaciones, banco y cooperativa escolar, de ajedrez.

c) Comité de: Trabajo (un representante por curso), disciplina, relaciones interescolares, programas y audiciones, ornato, biblioteca, finanzas, naciones unidas, viajes de estudio.

En síntesis, estas actividades eran completarías al contenido de los planes de estudio (común y variable) y además, representaban para los alumnos la oportunidad de cooperar con los organismos directivos del Liceo a través de actividades de libre desarrollo; de asumir responsabilidades importantes para la vida del Colegio; de adoptar iniciativas al servicio de una mejor convivencia social; de ejercer derechos; proyectar ideales, formarse normas valiosas de comportamiento, en una atmósfera de sana alegría, respeto, comprensión y tolerancia, más allá de los limites de la clase del curso.

Todas estas actividades han originado múltiples realizaciones cuya enumeración sería muy amplia para la síntesis que se ofrece en este capítulo. Por ello, se mencionará, a título de ejemplo, la ejecución de dos de estos proyectos, uno relativo a un programa de chilenidad realizado en el año 1941 y publicado en agosto de ese mismo año, cuyo texto contiene un resumen de actividades en pro de la chilenidad en las distintas asignaturas y otro, referente a los 20 años de la labor de la Corporación de Fomento de la Producción, y que se publicó en 1969, con el título "CORFO veinte años de labor".

\section{SISTEMA DE SEGURIDAD ESCOLAR.}

En noviembre del año 1966 se ensayó una operación evacuación y seguridad escolar, la que, por los logros obtenidos, se generalizó posteriormente a todos los 
establecimientos del país, esta experiencia recibió el nombre de "Operación Deyse" y con tal denominación se le conoce actualmente a nivel nacional.

Por la importancia que tiene se darán a continuación algunos antecedentes sobre su realización.

Sus fundamentos han sido basados en la urgente necesidad de que la población escolar conozca y practique las normas mínimas de seguridad aplicables en sus actividades diarias, a fin de evitar la improvisación y controlar las reacciones ante el peligro.

Su preparación duró tres meses y su realización días. Se escogió el liceo, por su calidad de experimental, por contar con el ciclo completo de Escuela primaria y secundaria, cuyo número de alumnos ascendía a 2500 aproximadamente y a la buena disposición de las autoridades y profesores del liceo.

En consecuencia, se procedió a la preparación de profesores y alumnos para actuar y atender, en caso de catástrofe, en forma intensa, permanente y científica.

Fue así como se estudiaron: saneamiento de los riesgos potencial de establecimiento, tanto de incendio como de estructuras del edificio; explicaciones para eliminar el pánico ante cualquier emergencia; planificación y gracias para evocaciones masivas por rutas y lugares seguros; confección de un plano de colegio para fijar las zonas de seguridad; formación de hábitos de disciplina ante la emergencia, etc.

Junto con la realización de los ejercicios de evocación, de las instrucciones y conferencias danzas a los alumnos y profesores, el cuerpo de hombres de Nuñoa actuaba con elementos reales, tales como alarmas internas y externas, apoyo aéreo, para lo cual un helicóptero aterrizó en uno de los patios del colegio para "evacuar heridos" y algo muy importante, se abrió una exposición sobre todos los posibles accidentes ocasionados por el fuego y su pretensión, la que visitaron autoridades y colegios de la comuna.

Las experiencias y enseñanzas de la "operación Deyse" fueron muy valiosas y a través de los años han tenido un reconocimiento oficial pues el Ministerio de Educación por circular no $782 / 79$ de 23 de junio de 1980 dio instrucciones para la aplicación del sistema de evaluación de los recintos y seguridad escolar de acuerdo a la "operación deyse".

\section{CALENDARIO ESCOLAR}

Este aporte se generalizó definitivamente en todos los establecimientos educacionales del país solo en la década del 70. 
Es por eso que en esta ocasión, se darán a conocer sus antecedentes más relevantes.

El liceo es un laboratorio en que, a través de experiencias cuidadosamente planeadas, se proporciona a los alumnos todas las oportunidades para que vivan en perfecta armonía con la naturaleza y se realice así su ideal de perfeccionamiento constante en un ambiente propio de comprensión y ayuda. Por esto el liceo debe dar a cada uno de sus miembros la oportunidad de desarrollar al máximo sus potencialidades, de donde surge una exigencia primordial: el planteamiento de la vida escolar, o sea, la necesidad de un calendario escolar elaborado en forma científica, de modo que, en cada etapa de la vida del alumno, se den todas aquellas tareas, actividades y experiencias que satisfagan sus necesidades físicas, intelectuales, emocionales y sociales.

El calendario escolar es un plan general que contiene la distribución de las actividades diarias y del año de toda la institución docente bien organizada. El calendario escolar comprende, pues, por una parte, las faenas relativas al horario diario de alumnos y de profesores, y por otra parte, todas las actividades que se extienden a lo largo del año escolar, que constituyen un complemento necesario de las primeras. Es por lo tanto, el medio más eficaz para procurar la buena administración de un colegio y proporciona, a la vez, una visión amplia y de conjunto del programa de las actividades escolares que debe realizar, a través del año, cada uno de los miembros de la comunidad docente: profesores, alumnos, autoridades educacionales, padres de familia.

De lo dicho se desprende, por un lado, la necesidad de que el calendario no sea confeccionado al comienzo del año lectivo y, por otro lado, que considere y consigne la totalidad de las actividades que se proyecta llevar a cabo en el curso del año docente; horarios de clases que integran los planes de estudio, consejos de cursos, consejos de los organismos técnicos, consejos parciales y generales de profesores, reuniones periódicas para estudiar, la situación escolar de los alumnos, horario destinado a actividades deportivas y de instituciones estudiantiles, calendarios referentes a la celebración de acontecimientos nacionales, patrióticos-históricos o relativos al propio colegio, fechas de exámenes de fin de curso, de repetición y de admisión, etc.

Es obvio que cada calendario escolar, para que cumpla con la finalidad a que está destinado, debe ajustare estrictamente a las condiciones y características de cada colegio comunidad y a las disposiciones que imparten las autoridades educacionales.

Los objetivos generales del calendario escolar pueden resumirse como sigue:

a) Dar a los profesores y a los alumnos la posibilidad de planear, organizar y aprovechar mejor el tiempo destinado al trabajo y a la creación. 
Cabe hacer notar a este respecto que el fracaso de muchos alumnos se ve con frecuencia, a la falta de un plan racional que les permita aprovechar y distribuir bien el tiempo.

Una tarea de gran interés pedagógico es precisamente la de averiguar de qué manera los alumnos distribuyen el tiempo relativo al trabajo y al juego, a sus tareas y estudios, con el fin de buscar soluciones prácticas a los problemas con que tropiezan en esta empresa.

b) Dar oportunidad a los profesores y a los padres de familia de hacer balances periódicos de las actividades estudiantiles y conocer los intereses reales y las actitudes sociales manifestados por los alumnos en el curso del desarrollo del calendario escolar.

c) Proveer al Colegio de un cuadro claro de sus actividades, a fin de que este arbitre los medios necesarios para su cumplimiento; en otras palabras, sirve de meta señera para la orientación de la labor docente anual de los diversos miembros que participan en la actividad escolar.

Para finalizar, se puede decir que gran parte de las conclusiones referentes a la importancia educacional del calendario escolar, se desprende de sus propios objetivos:

a) Desde luego, el calendario escolar, suministra a los alumnos y profesores una guía o método que permite distribuir mejor el tiempo de estudio y de trabajo.

b) Contribuye a que las personas encargadas de la misión de educar, realicen su trabajo en forma coordinada, consciente de las relaciones que deben existir, en la cada fase de la vida escolar, entre los principios fines formulados en la filosofía educacional respectiva y la realidad multiforme, cambiante y variada de los hechos constitutivos de la vida escolar.

c) Hace posible lograr un empleo provechoso del tiempo libre, ya que este podrá ser ocupado concretamente en la preparación y realización de algunas tareas específicas señaladas en el calendario.

d) Obliga, a quienes planifican el calendario, a preocuparse de la naturaleza de los intereses especiales de los alumnos, ya que ellos deben estar especialmente contemplados en todo calendario elaborado de forma científica. Por eso mismo, la elaboración de un calendario escolar contribuye, indirectamente, a que las personas encargadas de educar logren un mejor conocimiento de los intereses y necesidades del alumnado.

\section{CONSEJOS DE CURSO.}

Esta modalidad, tan especial en la vida escolar y que después de su creación y de un largo periodo de funcionamiento en el Liceo Manuel de Salas, fue adoptada 
por todos los colegios del país, ofrece en sus partes esenciales las siguientes características:

El consejo de curso es parte primordial de la vida escolar. Su finalidad básica es proponedora a la atención de las diferencias individuales de los alumnos. En este sentido, el consejo de curso puede desarrollar una vasta y fructífera labor, pues al proporcionar oportunidades de expresión de intereses y aptitudes del estudiante, esta institución estudiantil se convierte en uno de los más valiosos auxiliares para la exploración de gustos, inclinaciones y capacidades, y sus consecuentes orientación y cultivo.

Todos los aspectos esenciales en el desarrollo de la personalidad, encuentran en los consejos de curso estímulos y posibilidades de fomento. La aptitud para el trabajo en equipo, las condiciones organizadoras, los hábitos de responsabilidad y cumplimiento del deber, el trabajo honesto y la conducta sincera, la adhesión a los ideales y principios que forman la filosofía educacional del colegio, el espíritu alerta a los intereses y problemas de la comunidad, la estimación de las tradiciones y valores nacionales, la fraternidad y solidaridad humanas, etc., son rasgos que, a lo largo de los años, pueden tomar diversas formas de expresión y desenvolvimiento en los consejos de curso. Se comprende que, como instituciones educadoras que son, no caben en el marco de sus propósitos la vacía actividad académica de una sesión forma, la estéril discusión, por el afán de discutir, el caos disolvente o el descontrol. Tras el libre juego y la expresión de las necesidades e intereses más sentidos de los algunos, están la actividad de acucioso estudio, de firme serenidad, de inteligente orientación y dirección del profesor jefe. Es obvia, entonces, la importancia capital y decisiva de la acción directora y orientadora del profesor jefe.

El Liceo Manuel de Salas ha resumido en los siguientes puntos las finalidades de los consejos de curso:

a) Dar a los alumnos la oportunidad de formar ideales y actitudes que signifiquen un desarrollo progresivo de su personalidad y contribución al desenvolvimiento del grupo social en que actúan.

b) Proponedor a ampliar la cultura general de los educandos más allá de los límites de los programas escolares.

c) Capacitar a los estudiantes para ejercer derechos, cumplir obligaciones y asumir responsabilidades, por su participación directa y permanente en actividades individuales y de grupo.

d) Promover todas aquellas actividades que tiendan a proporcionar a los alumnos una mayor orientación educacional, de acuerdo con sus especiales capacidades, interés y aptitudes.

e) Crear un ambiente de recíproca comprensión entre profesores y alumnos. 
En cuanto a su organización, vale la pena señalar, en forma muy general, algunas normas al respecto. En la práctica, ellas deben reflejar lo más fielmente posible, los intereses y motivos de cada curso.

Desde luego, el consejo de curso deberá tener una mesa directiva. Sus autoridades podrían ser; Presidente, Vicepresidente, secretario, tesorero y alumno-jefe. Todas estas autoridades deben ser elegidas libre y democráticamente por los propios componentes de los cursos respectivos.

Deben surgir también de las propias manifestaciones del curso los diversos grupos o comités de trabajo. Ocioso sería insistir en la imposibilidad de obtener actividad alguna de comisiones impuestas, sin arraigo en algún interés o preocupación verdadera de los alumnos.

Los comités de trabajo podrían ser: de ornato y aseo, de cooperación social, de extensión cultural, que deben tener a su cargo actividades como diario mural, audiciones, charlas, bibliotecas de curso, correspondencia interescolar, comités de disciplina, comité de deportes.

Como norma básica, el profesor jefe deberá tener presente que el planteamiento del trabajo de los consejos de curso debe tener suficiente flexibilidad como para acoger cualquier iniciativa valiosa que pueda transformarse en una educativa empresa de grupo.

Las dificultades para el funcionamiento regular de los consejos de curso varían, naturalmente, de un curso a otro, de año en año, de ciclo en clico. No debería ser de otra manera puesto que se trabaja con material humano que es, de suyo, cambiante y en una etapa compleja del desarrollo de la personalidad. Por otra parte, surgen también otras dificultades derivadas de algunos factores negativos del medio familiar, tales como la falta de atención del hogar a los niños, frecuentes desorganización de este, excesiva libertad con que cuentan, muchas veces, los jóvenes y desconocimiento del buen empleo del dinero que les suministra el hogar, en ocasiones en forma pródiga. Agréguennos a esto, factores negativos del medio ambiente exterior: literatura de mala calidad o nociva, espectáculos poco educativos, propaganda comercial basada en afiches inadecuados para la niñez, etc. Todos estos factores plantean a la labor docente problemas que pueden parecer insolubles, pero cuyo estudio y tratamiento adecuado permiten, en gran porcentaje, el triunfo de la acción educadora que, en muchos casos, se torna verdaderamente dramática.

Los consejos de curso pueden, además, aportar importantes contribuciones en relación con el proceso mismo del aprendizaje y del rendimiento escolar. Encuestas preparadas por el profesor-jefe pueden dar ocasión a extensos y clarificadores debates acerca, por ejemplo, de: cómo se debe estudiar. La buena redacción, cómo ocupar mejor el tiempo libre, el arte de tomar apuntes etc. A modo de paréntesis, cabe anotar que, como es natural, los debates dan ocasión a los alumnos para 
mejorar su expresión oral y adquirir desenvoltura para hablar en público. Además, a través del consejo de curso y de la acción del profesor-jefe, pueden precisarse los motivos que dan origen a perturbaciones y deficiencias en el aprendizaje. En posición de los datos concretos al respecto el profesor-jefe puede solicitar, para buscarle solución, el concurso de los organismos pertinentes con que cuenta el liceo: servicios médico-dental, servicio de orientación, servicio de asistencia social y otros.

Finalmente, la existencia de los consejos de curso y de los profesores jefes que los supervigilan y orientan, permiten el establecimiento de un contacto permanente y efectivo entre el liceo y el hogar, relaciones estas que redundarán en innegable provecho en la tarea de la formación normal de la personalidad de los alumnos. En efecto, el consejo de curso suministra al profesor jefe oportunidades variadas para el conocimiento de algunos rasgos personales de los estudiantes, positivos o negativos, que con relativa frecuencia, ignoran los propios padres o apoderados. Por su parte el profesor jefe, en entrevistas con los padres o apoderados, podrá conocer otros rasgos que él, a su vez, ignoraba. Se comprende pues, que el intercambio de estos conocimientos entre el Liceo y el hogar tendrá un valor incalculable en la tarea formadora de la personalidad de los educandos.

Tales son, en apretado resumen, la naturaleza, finalidades y funcionamientos de los Consejos de Curso. Para una información más detallada, recomendaremos la lectura de un folleto publicado por el liceo Manuel de Salas, intitulado "Naturaleza y Funcionamiento de los consejos de curso".

\section{ORGANIZACIÓN ESTUDIANTIL}

Otro aporte a la educación secundaria fue la organización estudiantil o Centros de alumnos, tan comunes y generalizados ahora, en los liceos del país.

La organización máxima estudiantil era el consejo superior de alumnos y se organizó de acuerdo a las siguientes finalidades:

El consejo superior de alumnos está destinado a desarrollar una labor esencialmente educativa, a establecer las relaciones entre las diversas instituciones estudiantiles del colegio y a fiscalizar sus funciones. Procura formar conciencia en el alumnado acerca de la importancia de las instituciones estudiantiles en la vida escolar y en el cultivo de la personalidad de los alumnos y, promueve relaciones de carácter artístico, deportivo y cultural con instituciones afines de los demás liceos.

Sus reuniones tienen como objetivos primordiales los siguientes:

a) Unificar el pensamiento del alumnado en relación con los grandes acontecimientos de la vida ciudadana, con hechos sobresalientes de la cultura y con problemas que tengan especial significación para el desenvolvimiento de la personalidad de los alumnos o para la vida del colegio. 
B) Inspirar entre los alumnos del liceo, ideales y actividades valiosas para unas y eficiente convivencia social.

c) Ampliar y profundizar los intereses de los estudiantes y propender al desarrollo de la autoexpresión.

d) Arbitrar los medios para lograr un empleo útil de las horas libres o de descanso del alumnado.

e) Contribuir a la formación de hábitos correctos entre los alumnos a fin de que, en las diversas reuniones en que participen, se comporten como miembros de un auditorio inteligente, disciplinado y capaz, además de reconocer públicamente los éxitos merecedores de estímulo.

f) Correlaciones con las actividades e intereses del liceo y la comunidad.

Entre las preocupaciones del consejo superior de alumnos está expresamente excluido todo asunto de carácter político y religioso.

\section{ACTIVIDADES DE EXTENSIÓN CULTURAL}

Otro aporte a la educación secundaria, fue la extensión cultural, cuya característica, en el liceo experimental Manuel de Salas se indican en los párrafos siguientes:

a) Como complemento de su labor educativa, el liceo organiza planes de extensión cultura o de divulgación pedagógica, dentro y fuera del país, para los profesores, los alumnos, los padres y apoderados y el público en general. Coordina además, esta labor con la de las instituciones que realicen funciones similares.

b) La labor de la sección de extensión cultural se orienta, entre otras, hacia las siguientes actividades:

- organiza foros, ciclos de conferencias, cursos breves de extensión, conciertos, representaciones teatrales, programas de cine educativo, exposiciones artísticas y técnicas:

- auspicia campañas de salud y de alfabetización:

- Promueve concursos y otras actividades que tengan relación directa con el plan de extensión cultural; y

- Colabora con las autoridades de la comuna en su plan de extensión cultural, especialmente en proyectos que tiendan a un mejor conocimiento de la vida de la comuna.

\section{LAS INSTITUCIONES COOPERADORAS}

El liceo promovía la colaboración organizada de padres, apoderados, amigos y ex alumnos del colegio con el fin de buscar solución a los problemas de salud, 
socioeconómicos y educacionales de su alumnado; enriquecer la vida del liceo; extender su influencia a los distintos campos de la realidad social inmediata y confirmar y divulgar su labor educativa.

Para los efectos del cumplimiento de los objetivos antedichos, la dirección designaba anualmente un profesor que tenía su cargo la función de asesor de las instituciones cooperadoras del liceo.

El profesor asesor de las instituciones cooperadoras tenía las siguientes obligaciones y atribuciones:

Representar al liceo en las instituciones que colaboran o contribuyen al progreso del establecimiento:

Relacionar el liceo con la comunidad, a fin de hacer posible, entre ellos, una efectiva y recíproca comprensión y colaboración;

Relacionarse con la comisión de extensión cultural del liceo cuando alguna de las instituciones cooperadoras se proponga lleva a cabo tareas inherentes a la comisión referida; e

Informar permanentemente a la dirección sobre las diversas gestiones relativas a su cargo.

Entre las instituciones cooperadoras del liceo, podemos destacar las siguientes:

a) Asociación de padres y amigos del liceo

En el estudio y análisis de las características del liceo, se afirma que a su vida escolar se ha incorporado la opinión y colaboración de los padres y amigos del liceo. Creo la asociación de padres y amigos del liceo, la cual fue fundada en 1934 y su primer presidente fue don Pedro Godoy Pérez.

La asociación ha alcanzado un alto grado de desarrollo y su colaboración permanente a la vida escolar del colegio es un valioso medio para promover la solución de los múltiples problemas que afectan al niño y al adolescente, divulgar la labor educativa del liceo y desarrollar actividades de significación social para la comuna.

Esta organización que creó el liceo en la década del 30, empezó a extenderse paulatinamente a otros establecimientos del país desde los años 40 , hasta completarse definitivamente en el decenio del 60.

\section{b) Asociación de ex alumnos}

Si bien es cierto la asociación de ex alumnos, no fue original del liceo Manuel de Salas, en este tuvo una particularidad que lo hace único entres su congéneres.

La asociación de ex alumnos del liceo Manuel de Salas, tiene las siguientes finalidades:

- Continuar y divulgar la labor educativa del liceo.

- coordinar su actividad con la asociación de padres y amigos y de gobierno estudiantil. En cuanto a sus actividades, éstas eran las siguientes: 
- elaborar un plan de actividades para cooperar con el departamento de orientación e investigación en lo que se refiere a orientación educacional y vocacional.

- participar en la realización de los cursos de recuperación.

- fomentar el excursionismo en el alumnado.

- contribuir en la ejecución de nuevas obras de equipamiento e infraestructura del liceo.

- participar en las exposiciones y proyectos que realiza el liceo.

c) Fundación Manuel de Salas

La necesidad de emprender un plan de mejoramiento para el Liceo Experimental Manuel de Salas en el orden material, llevó a un grupo de padres, apoderados, ex alumnos y amigos del liceo a crear la Fundación Manuel de Salas, que obtuvo su personalidad jurídica en septiembre de 1963.

Las razones que se tuvieron en cuenta para crear la mencionada fundación fueron las siguientes:

- La necesidad de que el liceo Manuel de Salas cumpla con sus finalidades docentes experimentales y que se proyecte hacia la comunidad;

- La necesidad de dotar al liceo experimental Manuel de Salas de una biblioteca y un salón de actos que son indispensables para el desarrollo de su programa escolar, y

- La necesidad de buscar los medios para hacer frente a estos problemas ya que no es posible obtener los fondos de la universidad de Chile ni de otros organismos del Estado.

Esta fundación, gracias a la venta de bonos de cooperación educacional y de múltiples actividades pro-recaudación de fondos, logró, a través de la Sociedad Constructora de establecimientos Educacionales, construir un Aula Magna, que es un teatro con una capacidad superior a las 750 personas.

Las instituciones cooperadoras del Liceo Experimental Manuel de Salas, tienen una vitalidad única, debido a que se sienten incorporadas al proceso educativo del establecimiento y por ello, se han aplicado muy brevemente sus finalidades y objetivos a fin de que sirvan de modelo a otras instituciones de igual carácter que puedan crearse en otros establecimientos educacionales del país.

\section{ORDEN ESPECIAL DE RECONOCIMIENTO}

El liceo ha destacado y destaca a aquellos elementos mas relevantes de su propia comunidad y a otros, que, desde fuera, le han colaborado eficazmente en la solución de diversos problemas que ha tenido en los cincuenta años de existencia. 
Para ello, creó en el año 1936 la Orden Manuel de Salas cuyo lema es "Lealtad, Servicio y Progreso"; la condecoración respectiva se otorga tradicionalmente a los mejores alumnos que egresan del establecimiento y que hayan estudiado ininterrumpidamente en el colegio desde el nivel preescolar.

También se otorga la medalla respectiva a personalidades que hayan contribuido al engrandecimiento del liceo; de quienes la han recibido solo se nombrara a don Juvenal Hernández Jaque, cuatro veces Rector de la Universidad de Chile y Patrono del Liceo Manuel de Salas.

En esta apretada síntesis se ha mostrado la contribución que ha hecho de la Universidad de Chile, a través del liceo experimental Manuel de Salas, a la educación secundaria chilena.

Gran parte del aporte ya es realidad en los liceos de la república, lo que, sin contar con los medios materiales que el liceo Manuel de Salas recibió de la universidad, han podido, gracias a la mística del maestro, lograr resultados que permiten afirmar que las experiencias entregadas han contribuido, efectivamente, al fortalecimiento de la educación nacional.

Esta exposición de la tarea desarrolla por el Liceo Manuel de Salas, en las etapas de creación, búsqueda, ensayo y efectiva consolidación, ha sido objetiva y ceñida a documentos.

Las experiencias y las vivencias personales se han dejado fuera acaso por completo en un afán de mostrar, con equilibrio, lo que ha sido la labor de un equipo humano respaldado por una institución de visión generosa.

La Universidad de Chile acogió al liceo y lo hizo suyo. Este le dio a cambio, la solidez de un profesorado en permanente perfeccionamiento del cual la universidad tomó muchos elementos para servir o los compartió en el liceo, especialmente en su Facultad de Filosofía y Letras, Filosofía y Educación y por último, Facultad de Educación.

La experimentación en el Liceo no habría sido posible si no hubiese contado con la eficiente comunicación entre la cátedra universitaria y la del colegio; si no hubiese tenido la sólida presencia de sus académicos en las comisiones de concursos para proveer cargos; si los metodólogos de todas las asignaturas que formaban profesores con el prestigio de la Universidad de Chile no hubiesen hecho el Liceo Manuel de Salas, su laboratorio de ensayo pedagógico y su centro de prácticas docentes, de profesor jefe y de orientación; en fin, si no hubiese habido un nexo permanente y un contacto recíproco de nivel profesional con el Instituto Pedagógico y en especial y con la Rectoría y Servicios Centrales de la Universidad de Chile, en general.

Por muchos decenios, la Universidad de Chile fue la única, en el contexto hispano - americano, que contó con un liceo experimental propio, al servicio de la mejor formación de los docentes y de la educación nacional. 
También, muchos más países latinoamericanos recibieron el beneficio del aprendizaje permanente del profesor a través de los contratos a docentes del Liceo que llevaron experiencias del Liceo Manuel de Salas y de la Universidad de Chile a otros ámbitos.

La presencia de la Universidad de Chile fue una constante en todo quehacer del liceo y todo lo que éste logró lo hizo por esa tutoría espiritual amplia, que respaldaba sin presiones, y abiertas a todo saber como corresponde al concepto de universidad.

Esta crónica del trabajo señero del Liceo Manuel de Salas, es pues, con razón, la historia de cada maestro, de cada alumno, de todos los padres de los alumnos, de la comunidad entera, nacida, crecida y consolidada en la Universidad de Chile.

(17) República de Chile, Ministerio de Educación, 1932, Decreto nº 604.

* Todas las publicaciones tienen una completa Bibliografía de acuerdo a los temas tratados. 